\title{
Comparing Traditional Key Frame and Hybrid Animation
}

\author{
Eric Ahlström* \\ Blekinge Institute of Technology \\ Yooric90@hotmail.com
}

\author{
Lucas Holmqvist* \\ Blekinge Institute of Technology \\ lucas.holmqvist@gmail.com
}

\author{
Prashant Goswami \\ Blekinge Institute of Technology \\ prashant.goswami@bth.se
}

\begin{abstract}
In this research the authors explore a hybrid approach which uses the basic concept of key frame animation together with procedural animation to reduce the number of key frames needed for an animation clip. The two approaches are compared by conducting an experiment where the participating subjects were asked to rate them based on their visual appeal.
\end{abstract}

\section{CCS CONCEPTS}

- Computing methodologies $\rightarrow$ Procedural animation;

\section{KEYWORDS}

key frame animation, procedural animation, hybrid animation

\section{ACM Reference format:}

Eric Ahlström, Lucas Holmqvist, and Prashant Goswami. 2017. Comparing Traditional Key Frame and Hybrid Animation. In Proceedings of SCA '17, Los Angeles, CA, USA, fuly 28-30, 2017, 2 pages.

https://doi.org/10.1145/3099564.3106640

\section{INTRODUCTION}

The most common approach to creating animation clips is the key frame animation [Chen and Li 2016]. A powerful aspect of the key framing is the amount of control given to the artist but the downside is excessive memory consumption. A less common approach to character animation is procedural animation [Mahoney 1997] wherein the graphical artists only produce the model and then the animations are programmed separately. Procedural animation lends itself better to group behavior, secondary animation and inbetweening. We implement a hybrid animation approach which uses fewer key frames than the traditional key frame. It shifts a portion of the workload from the artists to the programmers but not to the extent of procedural animation [Rosen 2014]. The purpose of this study is to determine whether the hybrid animation can be considered as a preferable alternative to the traditional key frame animation approach.

\section{METHOD}

One of the crucial benefits of the hybrid animation is the low amount of key frames it requires. To this end, two key frames were extracted for each animation sequence and two additional key

\footnotetext{
${ }^{*}$ indicates equal contribution
}

Permission to make digital or hard copies of part or all of this work for personal or classroom use is granted without fee provided that copies are not made or distributed for profit or commercial advantage and that copies bear this notice and the full citation on the first page. Copyrights for third-party components of this work must be honored

For all other uses, contact the owner/author(s).

SCA '17, July 28-30, 2017, Los Angeles, CA, USA

(c) 2017 Copyright held by the owner/author(s).

ACM ISBN 978-1-4503-5091-4/17/07.

https://doi.org/10.1145/3099564.3106640 frames were generated from them using mirroring. When using four key frames to animate a clip with significant differences in rotation per key frame, the linear interpolation has poor visual quality. To avoid the sharp direction changes, a more sophisticated interpolation is needed that ensures continuous differentiability. We employed simple and efficient spherical spline quaternion interpolation called SQUAD [Shoemake 1985], see Figure 1. In several animations like crouching, the interpolations used in gait motions were not adequate. Additional extrapolation is required to cause the required oscillation around a keyframe, e.g. towards the end. A damped spring system was also added to the hybrid approach in some animations to remove the velocity jar, see Figure 2 .
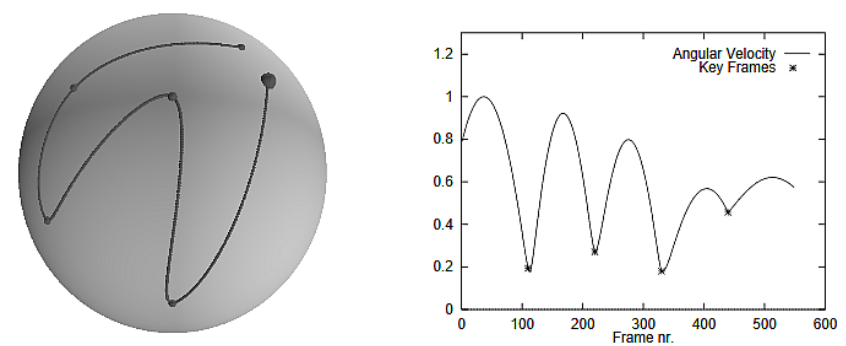

Figure 1: A sphere illustration of the spherical spline quaternion interpolation (SQUAD) and it's angular velocity graph, also see [Dam et al. 1998]

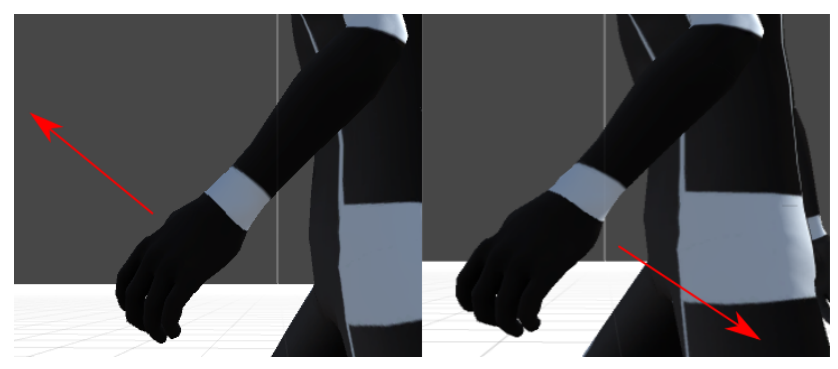

Figure 2: The abrupt change in the velocity direction of the hand between two consecutive frames causes velocity jar.

\section{RESULT}

The proposed system was implemented and tested on four animation clips: walking, running, crouching, and idling as displayed in Figure 3. The authors designed an experiment to find out how visually appealing the hybrid animation was compared side-by-side to the traditional key frame approach. Figure 4 summarizes results from 31 participants who compared and rated the clip according to 
what they found the most visually appealing. The rating was considered as negative if it favored the key frame animation significantly and positive if it favored hybrid animation slightly to significantly better. To increase the scalability of the implementation, support for increasing the amount of key frames used for the hybrid animation at intermediate points was added.

The participants were not informed of the technique used for the shown clips and the order of comparison was randomized to reduce the risk of learning bias. They were told to rate the clip according to what they found the most visually appealing (Likert scale with five possible answers). As the original key frame animation approach is based on motion capture data, the motions are expected to be more life-like and have high visual quality. This information is lost in the hybrid animation approach as there are not enough key frames to describe this behavior. On the other hand, the positive factors of reusable work and artist work relief could exceed the negatives in case of the hybrid method. In general, the response of the participants demonstrated that the hybrid animation was comparable to the key frame animation, see Figure 4.
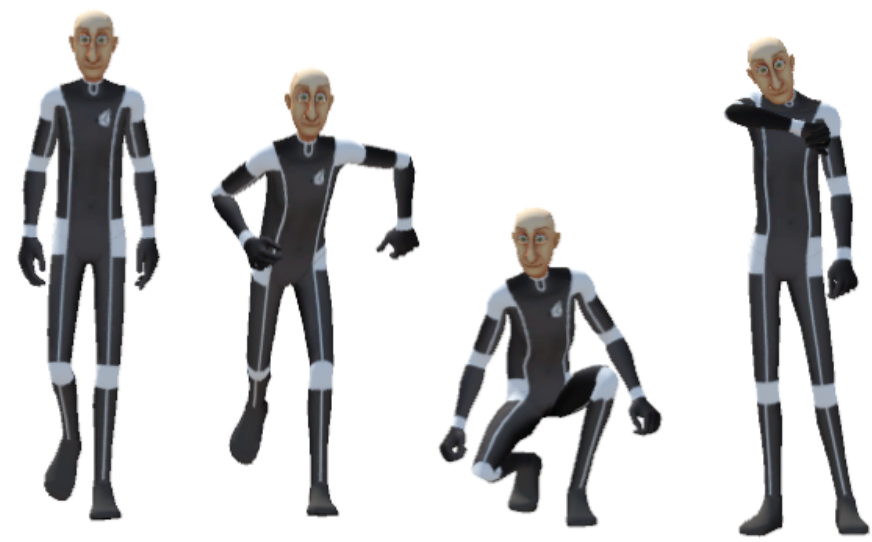

Figure 3: The four animation clips: walking, running, crouching and idling.

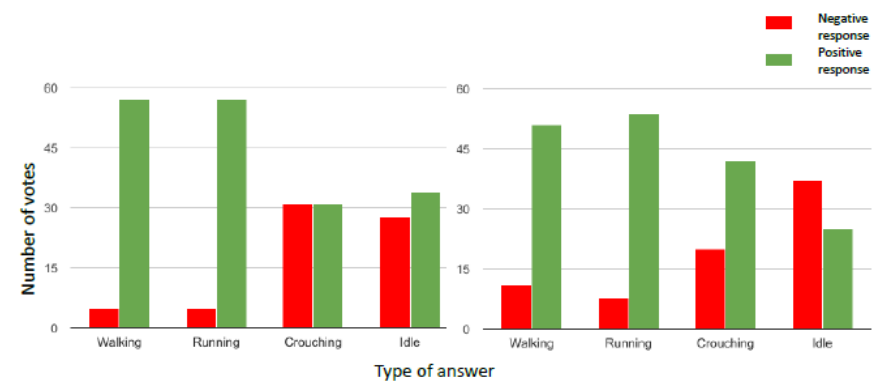

Figure 4: Participant ratings for hybrid animation in comparison to key frame approach with: (left) minimal keyframes, (right) extra keyframe(s) added.

\section{ACKNOWLEDGMENTS}

We would like to thank Vegard Myklebust, Ryan Juckett and Dave Hampson for providing source code that was used in our implementation.

\section{REFERENCES}

L. Chen and L. Li. 2016. Optimization of animation curve generation based on hermite spline interpolation. International fournal of Multimedia and Ubiquitous Engineering 11, 5 (2016), 337-344. www.scopus.com

B. Dam, E, M Koch, and M Lillholm. 1998. Quaternions, interpolation and animation. Technical Report.

D. P. Mahoney. 1997. Procedural animation. Computer Graphics World 20, 5 (1997), 39. http://miman.bib.bth.se/login?url=http://search.ebscohost.com/login. aspx?direct $=$ true $\& \mathrm{db}=\mathrm{afh} \& \mathrm{AN}=9705231610 \&$ site $=$ ehost-live

D. Rosen. 2014. Animation Bootcamp: An Indie Approach to Procedural Animation. http://www.gdcvault.com/play/1020583/ Animation-Bootcamp-An-Indie-Approach. (March 2014). http://www. gdcvault.com/play/1020583/Animation-Bootcamp-An-Indie-Approach

K Shoemake. 1985. ANIMATING ROTATION WITH QUATERNION CURVES. Computer Graphics (ACM) 19, 3 (1985), 245-254. www.scopus.com 